\title{
THEORY OF ELASTICITY AT THE NANO-SCALE
}

\author{
H.L. Duan ${ }^{1,2}$, J. Wang ${ }^{2}$ and B.L. Karihaloo ${ }^{3}$ \\ ${ }^{1}$ Institut für Nanotechnologie, Forschungszentrum Karlsruhe GmbH, 76021 Karlsruhe, Germany \\ ${ }^{2}$ LTCS and College of Engineering, Peking University, Beijing 100871, P. R. China \\ ${ }^{3}$ School of Engineering, Cardiff University, Queen's Buildings, The Parade, Cardiff CF24 3AA, UK
}

\section{Abstract}

We have shown in a series of recent papers that the classical theory of thermo-elasticity can be extended to the nano-scale by supplementing the equations of thermo-elasticity for the bulk material with the generalised Young-Laplace equations of surface elasticity. This talk will describe how this has been done in order to capture the often unusual thermo-mechanical and physical properties of nano-structured particulate and porous materials.

It will begin with a description of the generalised Young-Laplace equations for surface elasticity. We will then describe how the classical Eshelby formalism can be generalised to nanoinhomogeneities; unlike its classical counterpart the Eshelby tensor now depends on the size of the nano-inhomogeneity and the location of the material point in it. We will demonstrate its application on the calculation of the stress concentration factor of a spherical nano-void. We will next derive the Eshelby tensor for nano-particles consisting of a core surrounded by multiple outer shells. These multi-shell particles are used as novel functional materials as well as stiffeners/toughners in conventional composites and nano-composites. In these nano-heterogeneous particles, the mismatch of thermal expansion coefficients and lattice constants between neighbouring shells induces stress/strain fields in the core and shells, which in turn affect the physical/mechanical properties of the particles themselves and/or of the composites containing them. We will apply this solution to obtain the strain fields in quantum dots (QDs) with multi-shell structures and in alloyed QDs induced by the mismatch in the lattice constants of the atomic species.

The next part of the talk will address the generalisation of the micro-mechanical framework for determining the effective elastic properties and effective coefficients of thermal expansion of heterogeneous solids containing nano-inhomogeneities. We will use this generalised framework to calculate the effective elastic constants of nano-porous/cellular materials. It will be shown, in particular that these can be made to exceed those of the parent materials provided the pore surface elastic parameters satisfy certain conditions. These stiff nano-porous materials herald a radical breakthrough in sandwich-type construction. We will also use the generalised framework to study the thermo-elastic properties of heterogeneous materials containing spherical particles or cylindrical fibres. The interface between the matrix and second phase inhomogeneity is imperfect with either the displacement or the stress experiencing a jump across it. We will relate the effective coefficient of thermal expansion (CTE) to the effective elastic moduli and thereby generalise Levin's formula, and reveal two connections among the effective elastic moduli, thereby generalising Hill's connections. In contrast to the classical results, the effective CTE in the presence of an imperfect interface will be shown to be strongly dependent on the size of the inhomogeneity, besides the interface elastic and thermo-elastic properties.

The talk will end with the introduction of scaling laws governing the properties of nano-structured materials. The underlying cause of the size-dependence of these properties at the nano-scale is the competition between surface and bulk energies. These laws provide a yardstick for checking the accuracy of experimentally measured or numerically computed properties of nano-structured materials 
over a broad size range and can thus help replace repeated and exhaustive testing by one or a few tests.

\section{References}

[1] H.L. Duan and B.L. Karihaloo (2007). Thermo-elastic properties of heterogeneous materials with imperfect interfaces: Generalized Levin's formula and Hill's connections, J. Mech. Phys. Solids, 55, 1036-1052.

[2] H.L. Duan, B.L. Karihaloo, J. Wang and X. Yi (2006a). Strain distributions in nano-onions with uniform and non-uniform compositions, Nanotech., 17, 3380-3387.

[3] H.L. Duan, B.L. Karihaloo, J. Wang and X. Yi (2006b). Compatible composition profiles and critical sizes of alloyed quantum dots, Phys. Rev. B, 74, 195328-1-4.

[4] H.L. Duan, J. Wang, Z.P. Huang and B.L. Karihaloo (2005a). Eshelby formalism for nanoinhomogeneities, Proc. Roy. Soc. Lond. A, 461, 3335-3353.

[5] H.L. Duan, J. Wang, Z.P. Huang and B.L. Karihaloo (2005b). Size-dependent effective elastic constants of solids containing nano-inhomogeneities with interface stress, J. Mech. Phys. Solids, 53, 1574-1596.

[6] H.L. Duan, J. Wang, B.L. Karihaloo and Z.P. Huang (2006c). Nanoporous materials can be made stiffer than non-porous counterparts by surface modification, Acta Mater., 54, 2983-2990.

[7] J. Wang, H.L. Duan, Z.P. Huang and B.L. Karihaloo (2006). A scaling law for properties of nano-structured materials, Proc. Roy. Soc. Lond. A, 462, 1355-1363.

[8] J. Wang, H.L. Duan and B.L. Karihaloo (2007). Nano-mechanics or how to extend continuum mechanics to nanoscale, Bull. Pol. Acad. Sci., 55, 133-140.

[9] X. Yi, H.L. Duan, B.L. Karihaloo and J. Wang (2007). Eshelby formalism for multi-shell nanoinhomogeneities, Archives of Mech., 59, 259-282.

[10] H.L. Duan, J. Wang and B.L. Karihaloo, Theory of elasticity at the nano-scale, Adv. Appl. Mech., (in press). 